\title{
OPO LIDAR SOUNDING OF TRACE ATMOSPHERIC GASES IN THE 3 - 4 $\mu$ M SPECTRAL RANGE
}

\author{
Oleg A. Romanovskii ${ }^{1}{ }^{2 *}$, Sergey A. Sadovnikov ${ }^{1}$, Olga V. Kharchenko ${ }^{1}$, Semen V. Yakovlev ${ }^{1,2}$ \\ ${ }^{1}$ V.E. Zuev Institute of Atmospheric Optics, Tomsk, Russia, *roa@iao.ru \\ ${ }^{2}$ National Research Tomsk State University, Tomsk, Russia
}

\begin{abstract}
The applicability of a KTA crystal-based laser system with optical parametric oscillators (OPO) generation to lidar sounding of the atmosphere in the spectral range 3-4 $\mu \mathrm{m}$ is studied in this work. A technique developed for lidar sounding of trace atmospheric gases (TAG) is based on differential absorption lidar (DIAL) method and differential optical absorption spectroscopy (DOAS). The DIAL-DOAS technique is tested to estimate its efficiency for lidar sounding of atmospheric trace gases. The numerical simulation performed shows that a KTA-based OPO laser is a promising source of radiation for remote DIAL-DOAS sounding of the TAGs under study along surface tropospheric paths. A possibility of using a PD38-03-PR photodiode for the DIAL gas analysis of the atmosphere is shown.
\end{abstract}

\section{INTRODUCTION}

To overlap the near- and middle IR region, radiation from optical parametric oscillators based on nonlinear crystals is used [1]. However, laser radiation is required to be monochromatic in the standard DIAL technique. Finally, the problem of standard DIAL measurements at only two radiation wavelengths means that the disturbing absorption is ignored. The result of this is error introduced by a priori uncertain absorption coefficient. These disadvantages do not affect differential optical absorption spectroscopy (DOAS) [2]. However, DOAS capabilities for vertical profiling are restricted; only path-average measurements have been carried out until now. A technique which combines the advantages of both methods - spatial resolution of the DIAL technique and identification of gases in DOAScould be a promising approach to a solution of the problem. The aim of this work is the development of a technique for lidar sounding of trace atmospheric gases, which combines DIAL and DOAS, and its validation in a numerical experiment for estimating the capabilities of lidar sounding of the gas composition of the atmosphere in the 3-4 $\mu \mathrm{m}$ spectral range with an OPO-based laser system.

\section{DIAL AND DOAS TECHNIQUE FOR LIDAR SOUNDING}

The $P_{R}$ signal from a scattering layer of $\Delta z$ in thickness can be represented as

$P_{R}(z, \lambda)=P_{0}(\lambda) \frac{A_{D}}{z^{2}} \eta(\lambda) O(z) \Delta z \beta(z, \lambda) e^{-2 \tau(z, \lambda)}(1)$,

where $\mathrm{P}_{0}(\lambda)$ is the laser radiation power, $A_{D}$ is the instrumental function width, $O(z)$ is the overlapping region of the laser beam and detector's field-of view, $\beta(z, \lambda)$ is the mass coefficient of backscattering radiation, $\eta(\lambda)$ is the efficiency of the receiving-transmitting system, $\Delta z$ is the spatial resolution along the sounding path, and $\tau(z, \lambda)$ is the attenuation coefficient. The concentration of a gas under study is calculated by the DIAL equation

$$
n(z)=\frac{1}{2 \Delta \sigma_{a b s} \Delta z} \ln \left(\frac{P_{R}\left(z, \lambda_{o n}\right) P_{R}\left(z+\Delta z, \lambda_{\text {off }}\right)}{P_{R}\left(z, \lambda_{\text {off }}\right) P_{R}\left(z+\Delta z, \lambda_{o n}\right)}\right)
$$

where $\sigma_{\mathrm{abs}}$ is the absorption cross-section.

Disadvantages of the DIAL technique are caused by uncertainties of a priori absorption coefficients at two wavelengths. DOAS allows these disadvantages to be avoided, by monitoring the transmission in the UV, visible and IR regions in a broad band (20-100 nm). The first step in DOAS is the calculation of the ratio of the spectrum observed ( $\left.\mathrm{I}_{\mathrm{OBS}}\right)$ to the reference spectrum $\left(\mathrm{I}_{\mathrm{REF}}\right)$, which is found from the laser spectrum $\left(\mathrm{I}_{0}\right)$ measured with the same detector. An atmospheric spectrum at a known content of absorbing gases can be used as $I_{\text {REFF }}$. Calculating the ratio $\left(\mathrm{I}_{\mathrm{OBS}} / \mathrm{I}_{\mathrm{REF}}\right)$ and taking its natural logarithm, the optical depth can be found:

$-\ln \left(\frac{I_{O B S}}{I_{R E F}}\right)=\sum_{i} \sigma_{a b s}^{i}\left(\left[n_{i}(z)\right]_{O B S} \Delta z_{O B S}-\left[n_{i}(z)\right]_{R E F} \Delta z_{R E F}\right)+Y(\beta)$ 
Equation (3) includes the wavelength-dependent absorption (the first term in Eq. (3)) and the scattering $Y(\beta)$, which is mainly determined by the backscattering coefficient $\beta$. The ratio is independent of the laser radiation spectrum or the spectral dependence of the receiving optics, spectrometer, and detector, which is an important advantage of DOAS. The so measured magnitude is equal to the difference in the concentration of absorbing gases in the reference case and in the case of real atmosphere. A differential spectrum is usually retrieved by several hundreds of points; the number of fitting parameters is no more than six. Thus, equation (2) becomes overdetermined and is solved with the least-square method. Under this approach, the fitting coefficients are varied for the fitting spectrum to match the best with the spectrum observed. In this case, if the absorption coefficients are known, the integral content of each gas can be found.

Simultaneous determination of the concentrations of several gases is an important advantage of DOAS as compared to the DIAL technique.

\section{OPO LASER SYSTEM FOR REMOTE SOUNDING OF THE ATMOSPHERE}

In this work, we consider a laser system (designed by SOLAR LS Company, Minsk) which is a part of a OPO DIAL lidar and ensures the tunable generation of nanosecond radiation pulses in the 3-4 $\mu \mathrm{m}$ range. Tables 1 and 2 present main specifications of the pumping laser and radiation converter.

Table 1 Specifications of pumping laser

\begin{tabular}{|l|l|}
\hline Pulse frequency & $10 \mathrm{~Hz}$ \\
\hline Output energy: at $1064 \mathrm{~nm}$ & $350 \mathrm{~mJ}$ \\
\hline Pulse length at $1064 \mathrm{~nm}, \mathrm{FWHM}$ & $10 \ldots 13 \mathrm{~ns}$ \\
\hline Beam diameter at $1064 \mathrm{~nm}$ & $\leq 6 \mathrm{~mm}$ \\
\hline Beam divergence at $1064 \mathrm{~nm}$ & $\sim 1.5 \mathrm{mrad}$ \\
\hline Stability of pulse energy at $1064 \mathrm{~nm}$ & $\pm 2.5 \%$ \\
\hline
\end{tabular}

Table 2 Specifications of radiation converter

\begin{tabular}{|l|l|}
\hline Wavelength tuning range & $3 \ldots 4 \mu \mathrm{m}$ \\
\hline Radiation line width & $<5 \mathrm{~cm}^{-1}$ \\
\hline Pulse energy, in the tuning curve peak & $>5$ \\
\hline Pulse frequency & $10 \mathrm{~Hz}$ \\
\hline Radiation divergence & $</=2 \mathrm{mrad}$ \\
\hline Wavelength tuning control & with $3 \mathrm{SMs}$ \\
\hline
\end{tabular}

The DIAL-DOAS technique developed for TAG measurements was validated for estimation of the lidar signal levels, using the specifications of the above described KTA-based OPO laser system.

\section{SIMULATION OF TAG LIDAR MEASUREMENTS}

Sounding of some atmospheric gases $(\mathrm{HCl}, \mathrm{HBr}$, $\mathrm{NO}_{2}$, and $\mathrm{N}_{2} \mathrm{O}$ ) along surface atmospheric paths (in the range $1-5 \mathrm{~km}$ ) has been numerically simulated. The standard midlatitude summer model was used in the simulation. The absorption of all main atmospheric gases was considered; the $\mathrm{HCl}, \mathrm{HBr}$, and $\mathrm{NO}_{2}$ concentrations were taken equal to $1 \mathrm{ppm}$, and the $\mathrm{N}_{2} \mathrm{O}$ concentration, to 1.5 ppm. Table 3 represents the informative wavelengths used in the numerical simulation and appropriate for DIAL-DOAS sounding of the TAGs under study. Spatially and spectrally resolved lidar echo signals in the TAG wavelength range calculated for surface tropospheric paths are shown in Figs. 1a and $1 \mathrm{~b}$ for $\mathrm{N}_{2} \mathrm{O}$ and $\mathrm{NO}_{2}$, respectively, and in Figs. 2a and $2 \mathrm{~b}$, for $\mathrm{HBr}$ and $\mathrm{HCl}$, respectively.

Table 3 Informative wavelengths

\begin{tabular}{|c|c|c|c|c|}
\hline Gas & $\begin{array}{c}\lambda_{\text {abs }}, \mu \mathrm{m} \\
\text { (in air) }\end{array}$ & $\begin{array}{c}v_{\text {abs }}, \mathrm{cm}^{-1} \\
\text { (in air) }\end{array}$ & $\mathrm{T}_{\text {gas }}$ & $\mathrm{T}_{\text {dist }}$ \\
\hline $\mathrm{HBr}$ & 3.98730 & 2507.962 & 0.79 & 0.99 \\
\hline $\mathrm{HCl}$ & 3.57191 & 2799.622 & 0.60 & 0.93 \\
\hline $\mathrm{N}_{2} \mathrm{O}$ & 3.87664 & 2579.55 & 0.85 & 0.90 \\
\hline $\mathrm{NO}_{2}$ & 3.42577 & 2919.051 & 0.73 & 0.89 \\
\hline
\end{tabular}

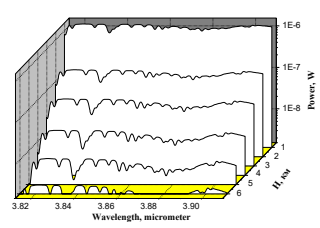

a)

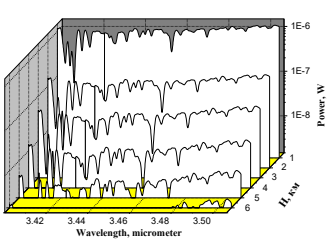

b)
Figure 1. Spatially and spectrally resolved lidar echo signals of (a) $\mathrm{N}_{2} \mathrm{O}$ and (b) $\mathrm{NO}_{2}$ sounding

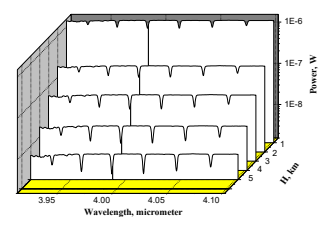

a)

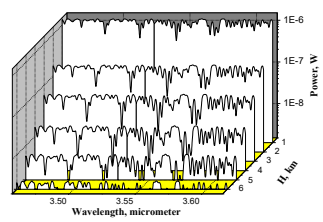

b)
Figure 2. Spatially and spectrally resolved lidar echo signals of (a) $\mathrm{HBr}$ and (b) $\mathrm{HCl}$ sounding 
Figures 1 and 2 show that the level of lidar echo signals exceed the level of photodetector noise equivalent power $\left(\mathrm{NEP}=10^{-9} \mathrm{~W}\right)$ in the whole altitude range under study $(0-5 \mathrm{~km})$.

The numerical simulation results show a possibility of retrieving lidar signals along paths up to $5 \mathrm{~km}$ long during sounding of $\mathrm{N}_{2} \mathrm{O}$ and $\mathrm{NO}_{2}$, as well as $\mathrm{HCl}$ and $\mathrm{HBr}$, with KTA-based OPO radiation in the $3-4 \mu \mathrm{m}$ range.

\section{MEASUREMENT RESULTS}

Energy parameters of a laser system have been measured in experiments with an OPO-based setup for estimation of optimal lidar output parameters. Two improved schemes for measuring output laser parameter are used.

In the first scheme, OPHIR PE10-C, PE25-C, and PE25-BB energy meters (EM) are used as the receiving unit.

A pumping wavelength of $1.064 \mathrm{~nm}$ is transformed by means of an optical parametric oscillator (OPO) based on nonlinear KTP and KTA crystals. Turning the crystal allows the radiation wavelength to be tuned in the 3-4 $\mathrm{mm}$ spectral range. A gold-coated end mirror allows the laser radiation to be directed to the EM detection area.

The measurements result in the wavelength dependence of the radiation pulse energy. In the second scheme, a gold-coated end mirror directs the laser radiation to a topographical target, which is represented by a diffusing reflector with an albedo of 0.8 . The main specifications of the setup are given in Table 4.

A sounding path is $20 \mathrm{~m}$ long. Backscattered radiation is gathered by a receiving Cassegrain telescope (main mirror diameter is $30 \mathrm{~mm}$ and focal length is $120 \mathrm{~mm}$ ) and focused to the PD3803-PR photodiode detection area. An aperture and BS-15 light filter are included in the scheme to prevent scattered radiation from saturating the photodiode.

The level of signals with the use of the above optical elements allows a suggestion about possibilities of sounding the atmosphere along a surface tropospheric path up to $1 \mathrm{~km}$ long.

The experimental geometry is shown in Figure 3. The time dynamics of a single radiation pulse (curve 1) and the shape of a sync pulse (curve 2) are shown in Fig. 4.
Table 4 Specification of the experimental setup

\begin{tabular}{|l|l|}
\hline Laser \\
\hline Wavelength turning range, $\mu \mathrm{m}$ & $3-4$ \\
\hline Maximal pulse energy, mJ & 9 \\
\hline Pulse length, ns & $10-13$ \\
\hline Pulse frequency, Hz & 10 \\
\hline Beam divergence, mrad & 2 \\
\hline Optical receiving system \\
\hline Main mirror diameter, mm & 300 \\
\hline Focal length, mm & 120 \\
\hline Analog-to-digital converter of signals \\
\hline Number of analog outputs & 2 \\
\hline Transmission band, MHZ & 200 \\
\hline Resolution, bit & 8 \\
\hline $\begin{array}{l}\text { Sampling frequency over all } \\
\text { channels, GS/s }\end{array}$ & 1 \\
\hline Photodetector & $1-4$ \\
\hline Spectral range, $\mu \mathrm{m}$ & 300 \\
\hline $\begin{array}{l}\text { Diameter of photodiode detection } \\
\text { area, } \mu \mathrm{m}\end{array}$ & 50 \\
\hline Input rise time, ns
\end{tabular}

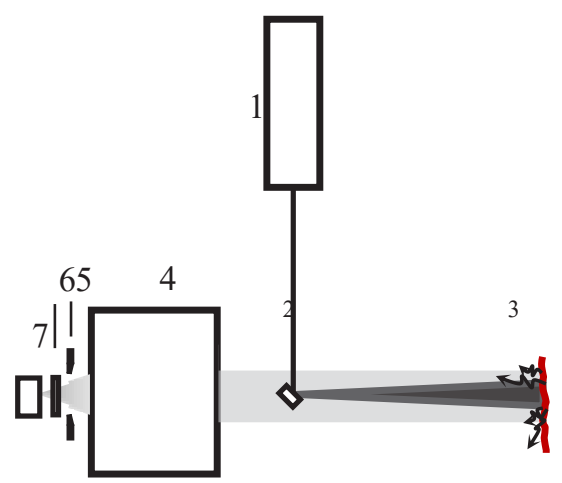

Figure 3 Geometry of the experiments: OPO-laser (1), mirror (2), diffusing reflector (3), receiving telescope (4), aperture (5), light filter (6), and photodiode (7)

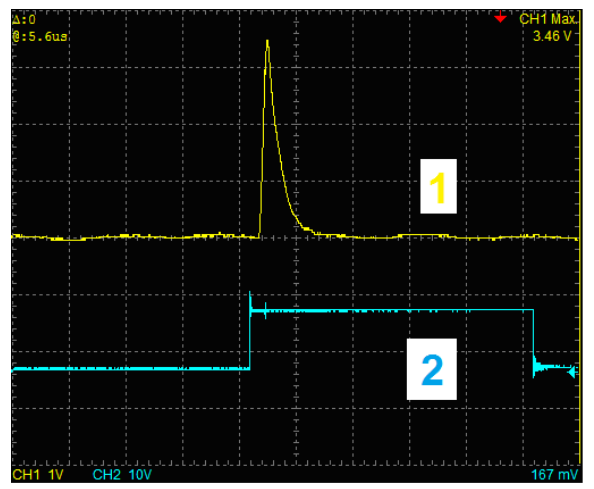

Figure 4 Time dynamics of a pulse of laser radiation at a wavelength of $3.335 \mu \mathrm{m}$ (1) and sync pulse shape (2) 
Figure $5-6$ shows energy parameters of the experimental setup measured in the first scheme with the use of EM and PD38-03-PR photodiode for path measurements with the receiving telescope. To check the correct operation of the laser throughout the wavelength tuning range, the pulse energy was measured with different EMs.

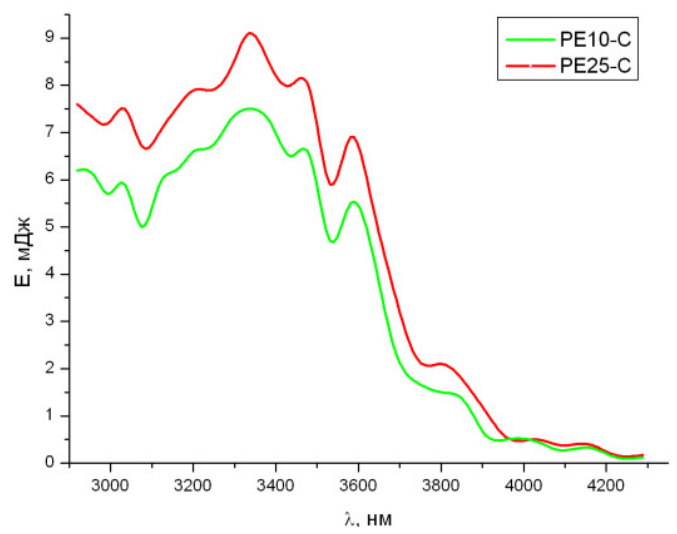

Figure 5 Pulse energy as a function of the radiation wavelength when using PE10-C and PE25-C EMs as radiation detectors

Figures $5-6$ shows the effect of water vapors on the lasing lines in the $4-\mu \mathrm{m}$ spectral region without nitrogen purging of the OPO cavity, i.e., the effect of strong water vapor absorption lines is obvious.

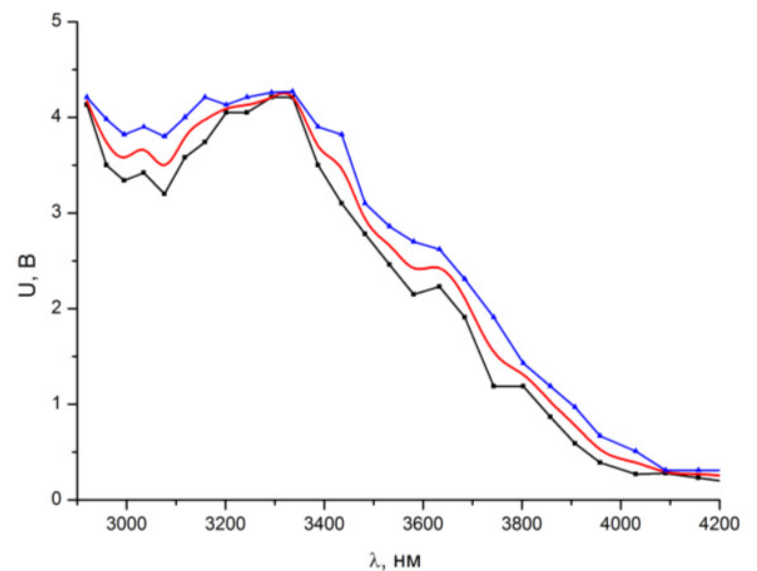

Figure 6 Lidar signals detected by the PD38-03-PR photodiode: minimum detectable lidar signals (black curve), maximum detectable lidar signals (blue curve), result of averaging a statistical sample of lidar signals (red curve)

\section{CONCLUSIONS}

The technique developed for lidar sounding of TAGs, which combines DIAL and DOAS, and its validation in numerical experiments confirm prospects of the use of the selected informative wavelengths. The numerical simulation performed shows that OPO laser is a promising source of radiation for remote DIAL-DOAS sounding of the TAGs under study along surface tropospheric paths. A possibility of using a PD38-03-PR photodiode for the DIAL gas analysis of the atmosphere is shown.

\section{ACKNOWLEDGEMENTS}

The work was supported by the President of the Russian Federation (grant no. MK-1367.2017.5 for the support of young Russian scientists).

\section{References}

[1] Mitev V., Babichenko S., Bennes J., 2013: Mid-IR DIAL for high-resolution mapping of explosive precursors, Proceeding of SPIE, 2013, 8894, 88940S.

[2] Platt U., Perner D., and Patz H. W., 1979: Simultaneous measurement of atmospheric $\mathrm{CH}_{2} \mathrm{O}, \mathrm{O}_{3}$, and $\mathrm{NO}_{2}$ by differential opticalabsorption, J. Geophys. Res, 84, pp. 63296335 . 